\title{
Mechanism of Particle Transport in Magnetized Silane Plasmas
}

S.-C. Yang, Y. Nakajima, Y. Maemura, Y. Matsuda and H. Fujiyama

Faculty of Engineering, Nagasaki University, 1-14 Bunkyo, Nagasaki 852, Japan

Short title : Particle Transport in Magnetized Silane Plasmas

\section{Abstract}

Particle transport phenomena were investigated in silane plasmas in the presence of magnetic field $B$ perpendicular to discharge electric field $E$. ¿From the experimental results, it was known that silicon particles were transported in the opposite direction of the $E \times B$ drift, and the particle density was decreased with increasing applied magnetic flux density. Theoretical calculations on particle drift show that negatively charged particles can be transported in the opposite direction of $E \times B$ drift and its drift velocity is increased with $B$ for the present experimental conditions. Both of experimental and theoretical results suggest that transport by modified ambipolar $E \times B$ drift can eliminate particles from discharge space. 


\section{Introduction}

Since the work of Spears et al. [1], particle generation has been observed in situations as different as etching plasmas [2], sputtering plasmas [3], and deposition plasmas [4 6]. The presence of particles (or dusty particles) in the plasma is a serious problem in etching, sputtering and deposition applications because of the substrate contamination. Recently studies of silicon particle generation and behavior are of increasing interest both from a scientific and technologic point of view, in the plasma enhanced chemical vapor deposition (PECVD) process for the deposition of a hydrogenated amorphous silicon (a-Si:H) thin film. In order to control the particle contamination in device fabrication applications, a thorough understanding of the correlation with forces that have an effect on particle behavior and spatial distribution in discharge space is required. Therefore, it has been found that electrostatic force, ion drag force, neutral drag force, gravitation force, thermophoretic force and polarization force are acting on the spatiotemporal distribution of silicon particles $[7,8]$.

However, at the present stage of the study of particle plasmas, little is known about the effects of a magnetic field perpendicular to the electric field, in DC silane plasmas. We have investigated dynamics of silicon particles in silane plasmas in the presence of a modulated magnetic field $B$ perpendicular to the discharge electric field $E[5$, 9, 10]. 
The goal of our study is to control the spatial distribution of the silicon particles and to eliminate them from the discharge space. In this article, we report the experimental results on the spatiotemporal evolution and the behavior of silicon particles in the presence of a magnetic field perpendicular to discharge electric field by observing two-dimensional profiles of spatially integrated Mie scattering intensity (SIMSI), and we compare them with calculation results based on the equation of motion for a three-component electronegative magnetized plasma.

\section{Experimental apparatus and methods}

\subsection{Experimental apparatus}

The schematic diagram of the experimental apparatus used in this study is shown in Fig. 1. The cylindrical vacuum chamber made of a stainless-steel was $230 \mathrm{~cm}$ in length and $32 \mathrm{~cm}$ in diameter. Two parallel approximate Rogowsky electrodes made of stainless steel (SUS304) were $90 \mathrm{~mm}$ in diameter and $30 \mathrm{~mm}$ in gap length. In the experiments, $10 \% \mathrm{SiH}_{4}$ gas diluted with $\mathrm{Ar}$ gas was used, and the gas pressures and gas flow rates were controlled by a mass flow controller and a capacitance manometer. Four solenoid coils were attached outside the vacuum chamber to produce homogeneous magnetic fields of $0 \sim 76$ Gauss in the axial direction of the chamber. 


\subsection{Particle detection}

In order to observe the spatiotemporal evolution $(i . \quad e$. , the generation and behavior) of dust particles in the discharge space, laser light scattering method was used. A He-Ne laser beam (5 $\mathrm{mW}$ at $632.8 \mathrm{~nm})$ and/or a pulsed dye laser pumped by a $\mathrm{XeCl}$ eximer laser ( $1 \mathrm{~mJ}$ at $488 \mathrm{~nm}$ in $20 \mathrm{~ns}$ duration) was alternatively used as the laser source for Mie scattering. Both laser beams were incident to the discharge space from a horizontal view port. The expended laser beam was shaped like a sheet $28 \mathrm{~mm}$ in height and $10 \mathrm{~mm}$ width using a cylindrical lens and an aperture. An image-intensified charge coupled device (ICCD) camera with a metal interference filter $(632.8 \mathrm{~nm}$ and/or $488 \mathrm{~nm}$ in center wavelength, $1 \mathrm{~nm}$ in full width at half maximum) was used to detect Mie scattering light by the silicon particles. The data obtained by the ICCD camera were digitized on the computer by 8 bit A/D converter and processed,giving a two-dimensional profile (contours) and the SIMSI. Since the Mie scattering intensity (MSI) is a complicated function of size, number density and refractive index of particles, the SIMSI represents only the overall feature of particles. In this study, the experiments were mainly performed for the conditions of the gas pressure $\mathrm{P}=0.3 \sim 0.4$ Torr and the discharge voltage $\mathrm{V}_{d}=400 \sim 600 \mathrm{~V}$. 


\section{Experimental results and discussion}

\subsection{Spatiotemporal evolution of two dimensional profile of particles}

Figure 2 shows the spatiotemporal evolution of MSI by the silicon particles under condition of pressure $\mathrm{P}=0.3$ Torr, discharge voltage $\mathrm{V}_{d}=500 \mathrm{~V}$ and magnetic flux density $\mathrm{B}=0$. From this figure, it is found that the MSI of silicon particles appears at about 10 minutes after the discharge start, and it increases with increasing discharge time and shows the tendency to saturate after 50 minutes. Figure 3 shows the temporal variation of the SIMSI (closed dot) obtained from the result of figure 2 and the discharge current (open dot). In this figure, the SIMSI and discharge current are normalized by the maximum value at the discharge time $\mathrm{V}_{t}=60$ minutes and the starting value at $\mathrm{V}_{t}=0$, respectively.

The results of figures 2 and 3, which indicate that it needs about 10 minutes to obtain the MSI by the silicon particles, results from the slow growth rate of the particles under this discharge condition. The SIMSI is increased dramatically because of the change from the initial nucleation to the coagulation stage. During the variation of the SIMSI, the discharge current increases to a maximum value at an early stage in the discharge time and decreases dramatically after the peak value, finally saturating with increasing discharge time. ¿From the above results, it is considered that the 
temporal variation of discharge current is caused by both the effects of the generation of silicon particles in the discharge space and the deposition of a-Si:H thin films on the cathode surface. In the early stage, discharge current has quite a small value caused by a decrease of electron density by the active nucleation process due to the electron sticking. Afterword, it increases by the saturation of electron sticking because of the change from the nucleation stage to the coagulation stage. Finally, it decreases and tends to saturate because of the deposition of a-Si:H thin films on the cathode surface [11]. The temporal variation of the discharge current qualitatively agrees with those of emission intensities from $\mathrm{SiH}^{*}(414.2 \mathrm{~nm})$ and $\mathrm{H}_{\alpha}(656.3 \mathrm{~nm})$. These results are connected with the temporal variation of the plasma resistance [12].

\subsection{Particle transport in the presence of magnetic field}

Figure 4 shows typical two-dimensional profiles of the MSI by silicon particles in the presence of a crossed magnetic field B perpendicular to the electric field E under the condition of pressure $\mathrm{P}=0.3$ Torr and discharge voltage $\mathrm{V}_{d}=500 \mathrm{~V}$. In this figure, the magnetic field is applied after the discharge time $\mathrm{V}_{t}=60$ minutes. In this time regime, plasma and silicon particles are in steady state even in a silane plasma, and then we can investigate only the effects of applied magnetic field. ¿From this figure, it is found that silicon particles were transported in the opposite direction 
of the $E \times B$ drift, and the particle density was decreased with increasing applied magnetic flux density. At high fields, the MSI by silicon particles disappeared from the discharge space.

In figure 5, the dependence of SIMSI data on applied magnetic flux density is shown for the condition of pressure $\mathrm{P}=0.3$ Torr. This figure shows that the silicon particle density is decreased with increasing applied magnetic flux density, and finally it is excluded from discharge space for the whole discharge voltage conditions. This particle motion in the crossed magnetic and electric fields will be explained as compared with theoretical calculations.

Figure 6 shows the dependence on discharge voltage of the magnetic flux density required to exclude silicon particles from the discharge space. ¿From this figure, it is found that in order to exclude silicon particles from the discharge space, a high applied magnetic flux density is needed with increasing pressure at high discharge voltages, but a low magnetic flux density is needed at low discharge voltages. This result is expected because the exclusion of silicon particles from the discharge space is due to the balance of the force of preservation $(i$. e. the balance of electrostatic force and ion drag force) and exclusion( $i$. e. neutral drag force), and these forces vary as discharge voltage and the pressure, respectively. 


\section{Theoretical calculation}

\subsection{Calculation model and basic equation}

In order to study the mechanism of particle drift motion, theoretical calculation has been performed with use of the equations of motion for the three-components; electrons, positive ions and negative ions, $i$. e., negatively charged heavy particles. In this calculation, we assume electric field $E=\left(E_{x}, 0,0\right)$ and homogeneous magnetic field $B=\left(0,0,-B_{z}\right)$ as shown in figure 7 . Thus the $E \times B$ drift of plasma occurs in the $y$-direction in the figure 7 . Considering only the components perpendicular to $B$, the equation of motion including collisions is obtained as follows [13],

$$
m n \frac{d v_{\perp}}{d t}= \pm q n\left(E+v_{\perp} \times B\right)-k T \nabla n-m n \nu v_{\perp}+F
$$

where the \pm and $F$ indicate the sign of the charges and the external force density acting on the spatial distribution of particles, respectively. In the steady state plasma, the inertia term can be neglected. In equilibrium the sum of the average currents must balance;

$$
\sum_{j=e, i, p} q_{j} \Gamma_{j}=0
$$


here $\Gamma_{j}=-D_{j} \nabla n_{j} \pm n_{j} v_{j}$ is a flux equation. Thus the current balance in the $y$ direction is

$$
\begin{aligned}
e \Gamma_{e y}+e \Gamma_{i y}+Q_{n} e \Gamma_{p y}= & -e n_{e} v_{e y}-Q_{n} e n_{p} v_{p y}+e n_{i} v_{i y} \\
& -e D_{e} \nabla n_{e y}+e D_{i} \nabla n_{i y}-Q_{n} e D_{p} \nabla n_{p y} \\
= & 0 .
\end{aligned}
$$

Here we neglect the density gradient in the $y$-direction,

$$
-n_{e} v_{e y}-Q_{n} n_{p} v_{p y}+n_{i} v_{i y}=0
$$

To take electron attachment and detachment effects into account, we introduce the charging rate $\beta=n_{p-} / n_{p}$; here $n_{p}=n_{p-}+n_{p 0}$ is the ratio of the number density of charged particles, $n_{p-}$, to total particle number density $n_{p}$, and $n_{p 0}$ is a neutral particle density in the present calculation. It is assumed that the neutral particles are generated, because negatively charged particles are impinged by energetic electrons accelerated by the sheath voltage and then the secondary electrons are emitted from the particles. So the charge neutrality condition should be modified as follows;

$$
n_{e}+\beta Q_{n} n_{p}=n_{i}
$$

Using the balance of average current in equilibrium, eq. (??), and the charge neutrality condition, eq. (5), we can obtain the space-charge electric field $E_{y}$ and drift 
velocities $v_{e y}, v_{i y}$ and $v_{p y}$. The drift velocities of each species are obtained as follows;

$$
\begin{aligned}
v_{e y} & =-\mu_{e \perp} E_{y}-D_{e \perp} \frac{1}{n_{e}} \frac{\partial n_{e}}{\partial y}+\frac{h_{e}^{2}}{1+h_{e}^{2}}\left(v_{E \times B}+v_{e D y}\right)+\frac{1}{1+h_{e}^{2}} \frac{1}{n_{e} m_{e} \nu_{e n}} F_{y}, \\
v_{i y} & =\mu_{i \perp} E_{y}-D_{i \perp} \frac{1}{n_{i}} \frac{\partial n_{i}}{\partial y}+\frac{h_{i}^{2}}{1+h_{i}^{2}}\left(v_{E \times B}+v_{i D y}\right)+\frac{1}{1+h_{i}^{2}} \frac{1}{n_{i} m_{i} \nu_{i n}} F_{y}, \\
v_{p y} & =-\mu_{p \perp} E_{y}-D_{p \perp} \frac{1}{n_{p}} \frac{\partial n_{p}}{\partial y}+\frac{h_{p}^{2}}{1+h_{p}^{2}}\left(v_{E \times B}+v_{p D y}\right)+\frac{1}{1+h_{p}^{2}} \frac{1}{n_{p} m_{p} \nu_{p n}} F_{y},
\end{aligned}
$$

where these subscripts $e, i$ and $p$ refer to electrons, positive ions and negatively charged particles, respectively. Here $v_{E \times B}$ is the $E \times B$ drift velocity, $\mu=\frac{e}{m \nu}$ is the mobility,$\nu=\frac{v_{t h}}{n \sigma}$ is the particle collision frequency which neutrals, $v_{D y}=$ $\frac{K T}{e B} \frac{n^{\prime}}{n}$ is the diamagnetic drift velocity, $h=\frac{\omega_{c} \tau}{2 \pi}$ (cyclotron frequency $\omega_{c}$, collision time $\tau$ ) is a Hall parameter and $F_{y}$ represents the other forces.

The time rate of change of particle charge is related to the currents of ions $I_{i}$ and electrons $I_{e}$ that impinge on the particle $(i . e .$, particles are considered as spherical probes). Because the particles are isolated, they behave like floating probes resulting in a negative potential. The electron and ion currents are well known for spherical probes in a Maxwellian plasma [14] :

$$
\begin{aligned}
& I_{i}(\phi)=4 \pi r_{p}^{2} \frac{e n_{i}}{4}\left(\frac{8 K T_{i}}{\pi m_{i}}\right)^{1 / 2}\left(1-\frac{e \phi}{K T_{i}}\right), \\
& I_{e}(\phi)=-4 \pi r_{p}^{2} \frac{e n_{e}}{4}\left(\frac{8 K T_{e}}{\pi m_{i}}\right)^{1 / 2} \exp \left(\frac{e \phi}{K T_{e}}\right),
\end{aligned}
$$


where $\phi$ is the surface potential of the particle, and $n_{i}\left(n_{e}\right), T_{i}\left(T_{e}\right)$ and $m_{i}\left(m_{e}\right)$ are the ion (electron) density, temperature and mass, respectively.

In this model the floating potential is determined by the relation $I_{i}+I_{e}=0$. Therefore, the charge of the particles is only a function of the particle radius, because the floating potential is also only a function of the radius.

\subsection{Calculation Results and Discussion}

Figure 8 shows typical calculation results of drift velocities of (a) electrons, $v_{\text {ey }}$, and ions, $v_{i y}$, and (b) negatively charged particles, $v_{p y}$, for a variation of particle radius $r_{p}$ and electron Hall parameter $h_{e}$. In this calculation, we assumed various parameters as follows; electric field $E=3 \times 10^{3} \mathrm{~V} / \mathrm{m}$, gas pressure $P=0.3$ Torr, plasma density $n_{i}=n_{e 0}=1 \times 10^{16} \mathrm{~m}^{-3}$, electron temperature $k T_{e}=1 \mathrm{eV}$, ion and particle temperatures $k T_{i}=k T_{p}=0.025 \mathrm{eV}$, room temperature, mass density of particles $\rho=2.2 \times 10^{3} \mathrm{~kg} / \mathrm{m}^{3}$, number density of particles $n_{p}=10^{13} \mathrm{~m}^{-3}$, charging rate $\beta=1$, mass of positive ion $m_{i}=6.692 \times 10^{-26} \mathrm{~kg}$, collision frequency of electron, positive ion and negative ion $\nu_{e}=3.88 \times 10^{7} \mathrm{sec}^{-1}, \nu_{i}=2.16 \times 10^{6} \mathrm{sec}^{-1}$ and $\nu_{p}=5.83 \times 10^{-9} \times r_{p}{ }^{-\frac{3}{2}} \sec ^{-1}$, respectively.

In this figure, particles have negative velocity, which means particles are transported in the opposite direction of the $E \times B$ drift, $v_{e y}$ and $v_{i y}$. The drift velocities of particles and ions are decreased with increasing particle radius $r_{p}$. But the electrons drift 
velocities increase with $r_{p}$. This can be explained by the decrease of total ambipolar electric field among three species. Namely, in weakly magnetized plasmas consisting of electrons and ions, since only electrons move by $E \times B$ drift, a space charge electric field is formed due to heavy non-magnetized ions. Then both electrons and ions are transported by this space charge electric field at the same drift velocity which is slower than $V_{E \times B}=E / B$. This type of $E \times B$ drift is an ambipolar $E \times B$ drift. However, when heavy particles with attached electrons are present in the plasma, the space charge electric field is weakened by non-drifting negative particles and reduced with electrons. As a result of the reduced electric field, electrons drift faster and ions slower than those in a plasma without particles. Then negatively charged particles move slowly in the direction opposite to the $E \times B$ drift.

On the other hand the drift velocities of particles, ions and electrons are increased with increasing electron Hall parameter $h_{e}$. With increasing $h_{e}$, i.e., crossed magnetic field $B$, the value of $v_{p y}$ is increased for weak $B$ and is decreased for strong $B$. This can be explained by the fact that the strength of the space charge electric field depends on the magnetic flux density. Namely, for strong $B$ the space charge electric field is weakened by the $E \times B$ drift of ions. For conditions of large $r_{p}$ or low $n_{e}$, the electrons move faster, but both ions and particles move slower due to the reduced space charge electric field. These calculation results agree with the experimental results. 
¿From these calculation results, it is found that $v_{\text {ey }}=3.2 \times 10^{3} \mathrm{~m} / \mathrm{s}, v_{i y}=2.6 \times 10^{3}$ $\mathrm{m} / \mathrm{s}$ and $v_{p y}=40 \mathrm{~m} / \mathrm{s}$ for the conditions of $r_{p}=100 \mathrm{~nm}, h_{e}=5$ and $\mathrm{P}=0.3$ Torr, i.e., the experimental conditions. ¿From the above experimental and theoretical results, it is suggested that modified ambipolar $E \times B$ drifts can eliminate particles from the discharge space.

\section{Conclusion}

In DC silane plasmas, silicon particles were generated and transported in the direction opposite to the $E \times B$ drift, and the spatially integrated Mie scattering intensity (SIMSI) was decreased with increasing applied magnetic flux density.

The experimental results on profiles of $\mathrm{SiH}^{*}$ radicals, plasmas and MSI in the cross magnetic field supports the hypothesis that useful radicals for deposition process can be separated from harmful silicon particles by the crossed magnetic field. When the applied magnetic field was modulated by a low frequency signal, the particles moved in the direction opposite to the $E \times B$ drift, more slowly as compared with the plasma motion. In this case, a more drastic decrease of SIMSI was observed. This suggests that the particles are slowly transported by the modified space charge effect caused by the $E \times B$ drift.

These experimental results qualitatively agree with the calculation results based on 
the equations of motion for a three-component electronegative plasma.

\section{Acknowledgments}

This work was supported partly by a Grant-in-Aid for Scientific Research on Free Radical Science in Priority Areas by Ministry of Education, Science and Culture. It is a pleasure to thank M. Morita of Nagasaki university for his technical assistance. 


\section{References}

[1] K. G. Spear, T. J. Robinson and R. M. Roth, 1986 IEEE Trans. Plasma Sci. PS-14 179

[2] G. S. Selwyn, J. Singh and R. S. Bennett 1989 J. Vac. Sci. Technol. A7 2758

[3] G. S. Selwyn, J. S. Killop, K. L. Haller and J. J. Wu 1990 J. Vac. Sci. Technol. A8 6

[4] Y. Watanabe, M. Shiratani and M. Yamashita 1992 Appl. Phys. Lett. 61 1520

[5] L. Boufendi and A. Bouchoule 1994 Plasma Sources Sci. Technol. 3262

[6] H. Fujiyama, H. Kawasaki, S. -C. Yang and Y. Matsuda 1994 Jpn. J. Appl. Phys. 334216

[7] M. S. Barnes, J. H. Keller, J. C. Forster 1992 J. A. O’Neill and D. K. Coultas Phys. Rev. Lett. 68313

[8] D. Winske and M. E. Jones 1994 IEEE Trans. Plasma Sci. 22454

[9] H. Fujiyama, T. Yamashita, T. Takahashi and H. Matsuo 1987 Appl. Phys. Lett. 501322

[10] H. Fujiyama, H. Kawasaki, Y. Matsuda and N. Ohno 1991 Mater. Sci. Eng. A140 569

[11] S.-C. Yang, Y. Matsuda and H. Fujiyama 1993 Proc. 6th Symp. on Plasma Science for Materials 21 
[12] S.-C. Yang, Y. Matsuda and H. Fujiyama 1994 Proc. 2nd Asia-Pacific Conf. on Plasma Sci. \& Technol. 204

[13] F. F. Chen 1984 Introduction to Plasma Physics and Controlled Fusion (Plenum Press) Chapter 5

[14] T. Trottenberg, A. Melzer and A. piel 1995 Plasma Sources Sci. Technol. 4 450 


\section{Figure Captions}

Fig. 1 Experimental apparatus.

Fig. 2 Spatiotemporal evolution of MSI caused by the silicon particles.

Fig. 3 Temporal variations of the SIMSI and discharge current versus discharge time.

Fig. 4 Two-dimensional profiles of MSI caused by the silicon particles in the presence of a magnetic field $B$ perpendicular to the electric field $E$.

Fig. 5 Dependence of SIMSI data on applied magnetic flux density.

Fig. 6 Dependence on discharge voltage of the magnetic flux density required to exclude silicon particles from the discharge space.

Fig. 7 Calculation model in a DC dusty plasma in the presence of a crossed magnetic field.

Fig. 8 Typical calculation results of drift velocity of (a) electrons and ions, and (b) negative ions (silicon particles) for particle radius $r_{p}$ and electron Hall parameter $h_{e}$. 


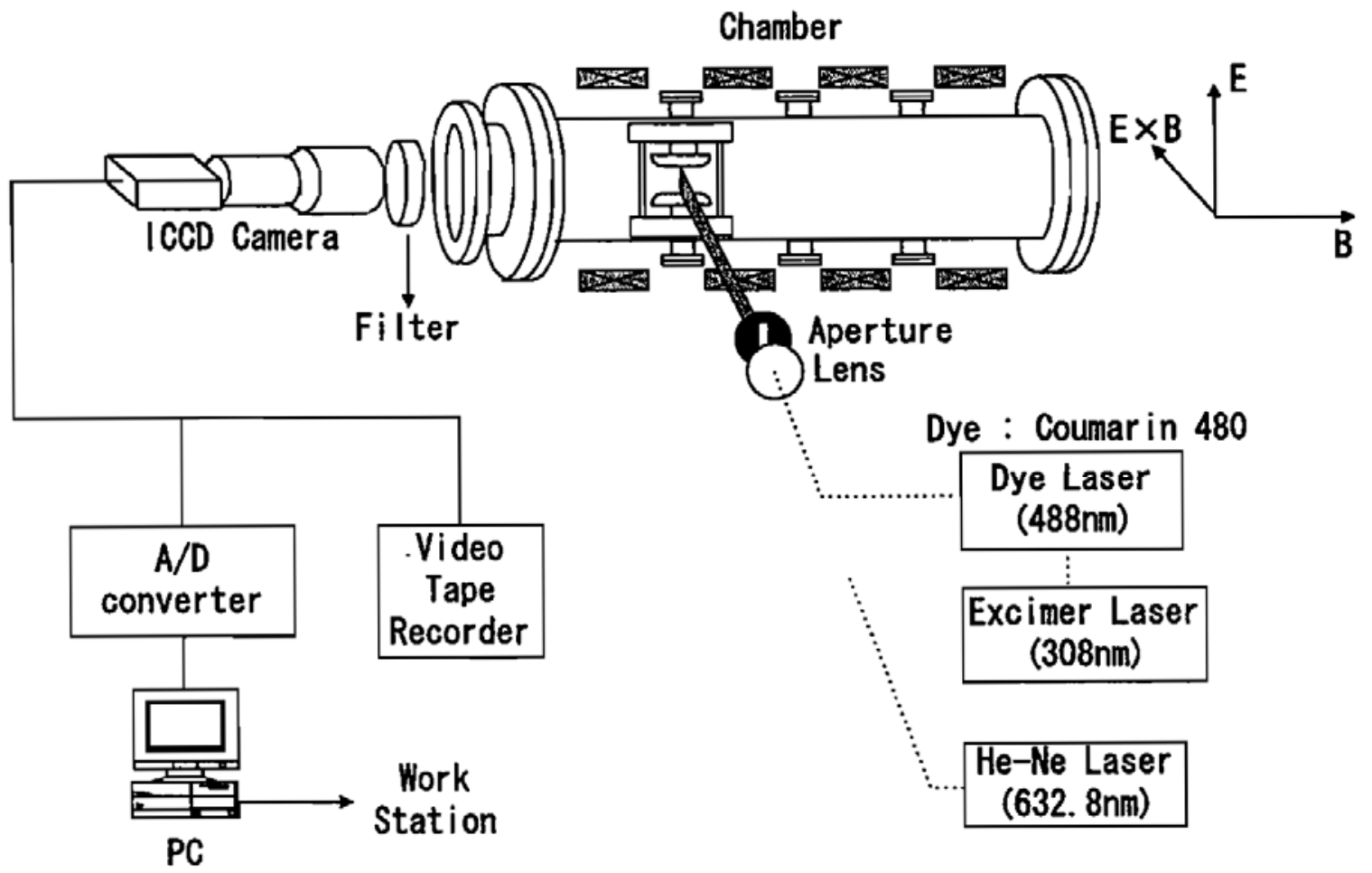

Figure 1. Experimental apparatus. 

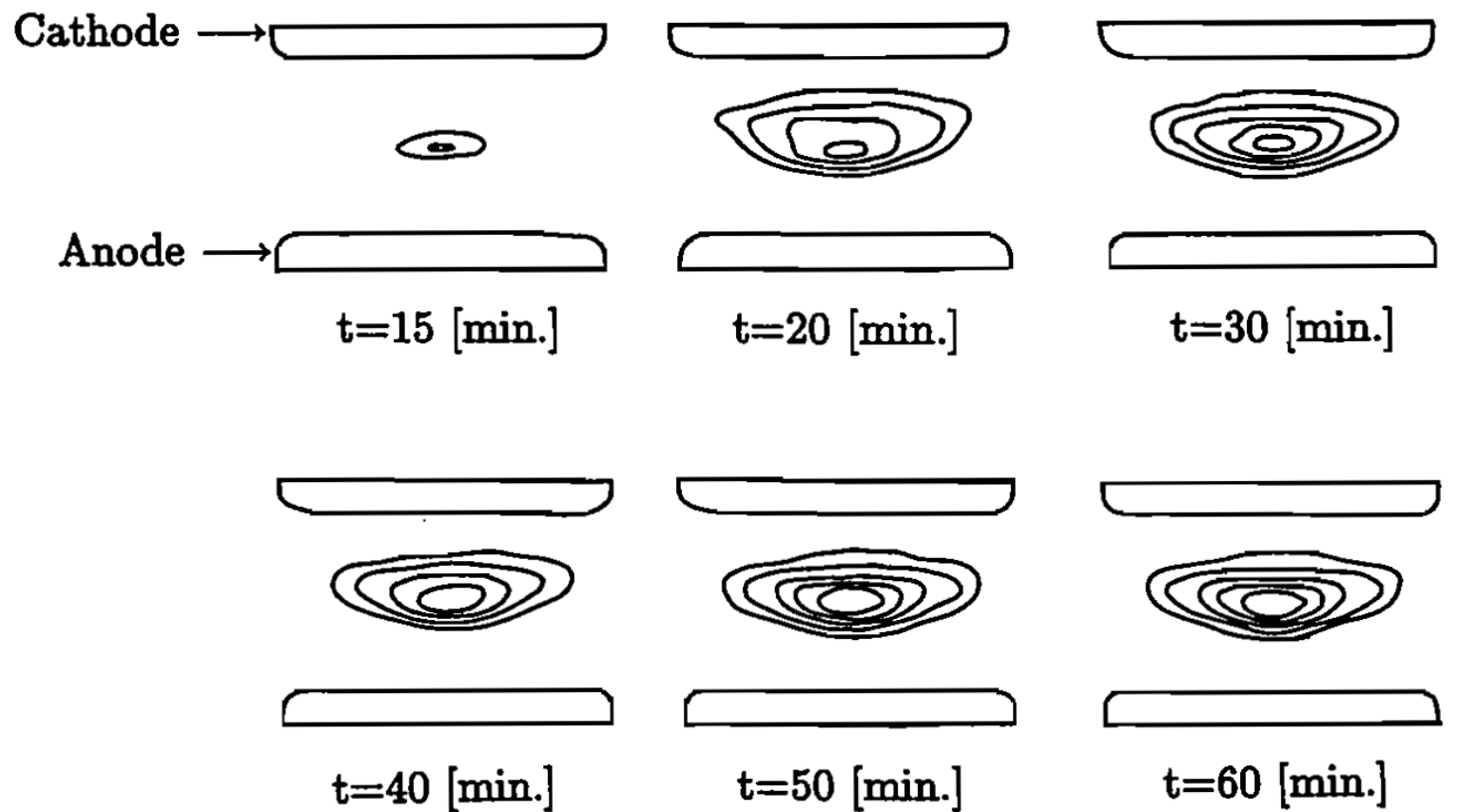

$$
\mathrm{P}=0.3 \text { [Torr], } \mathrm{V}=500[\mathrm{~V}]
$$

Figure 2. Spatiotemporal evolution of MSI caused by the silicon particles. 


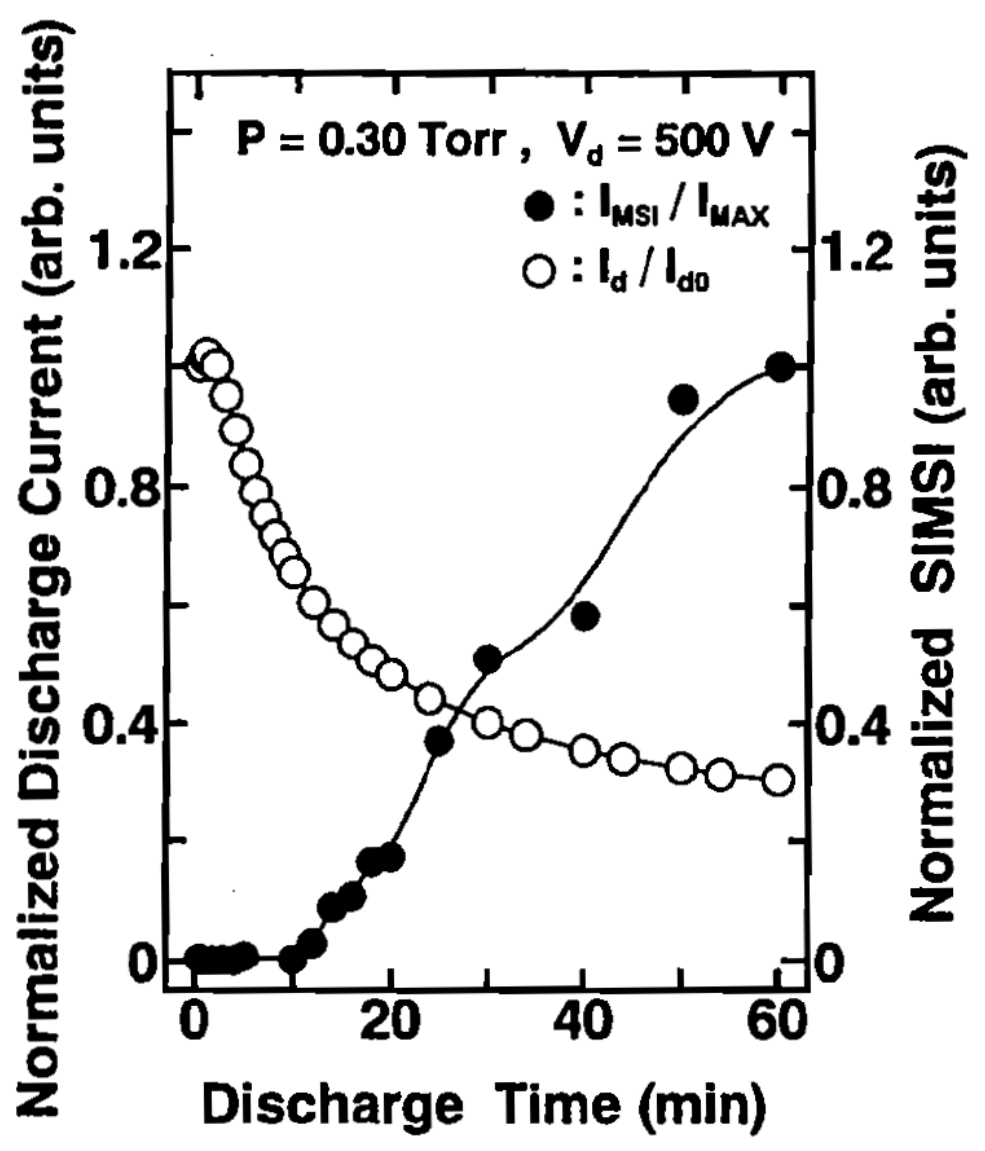

Figure 3. Temporal variations of the SIMSI and discharge current versus discharge time. 

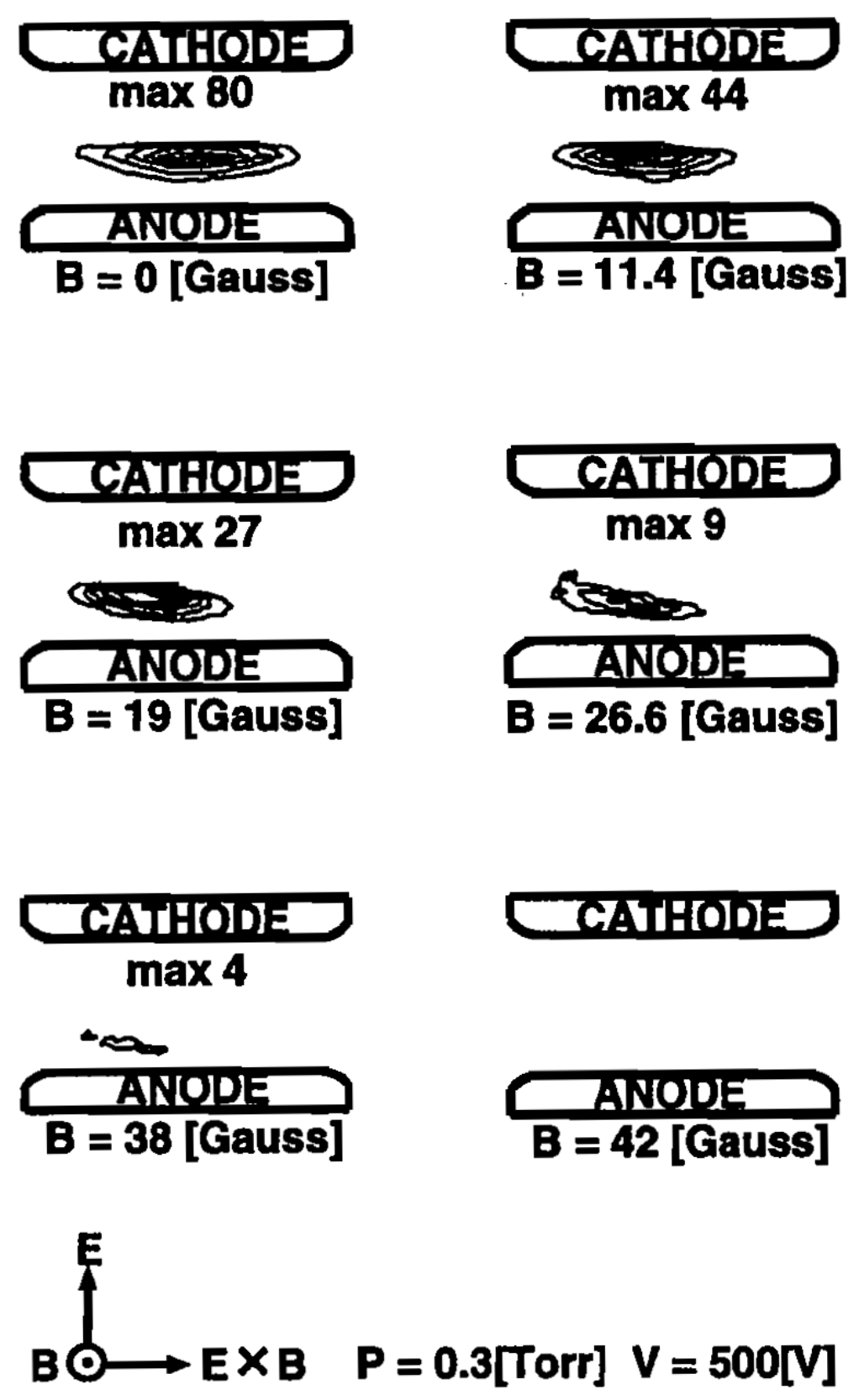

Figure 4. Two-dimensional profiles of MSI caused by the silicon particles in the presence of a magnetic field $B$ perpendicular to the electric field $E$. 


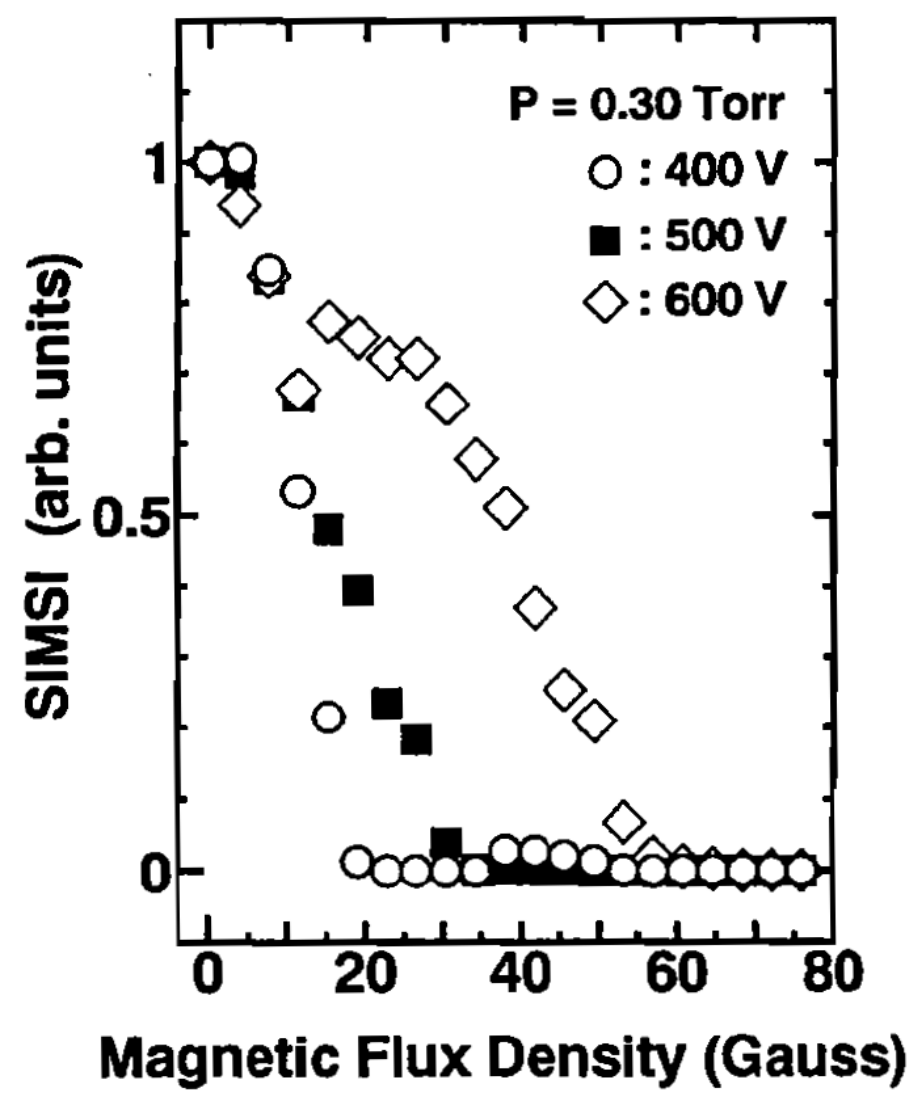

Figure 5. Dependence on SIMSI data on applied magnetic flux density. 


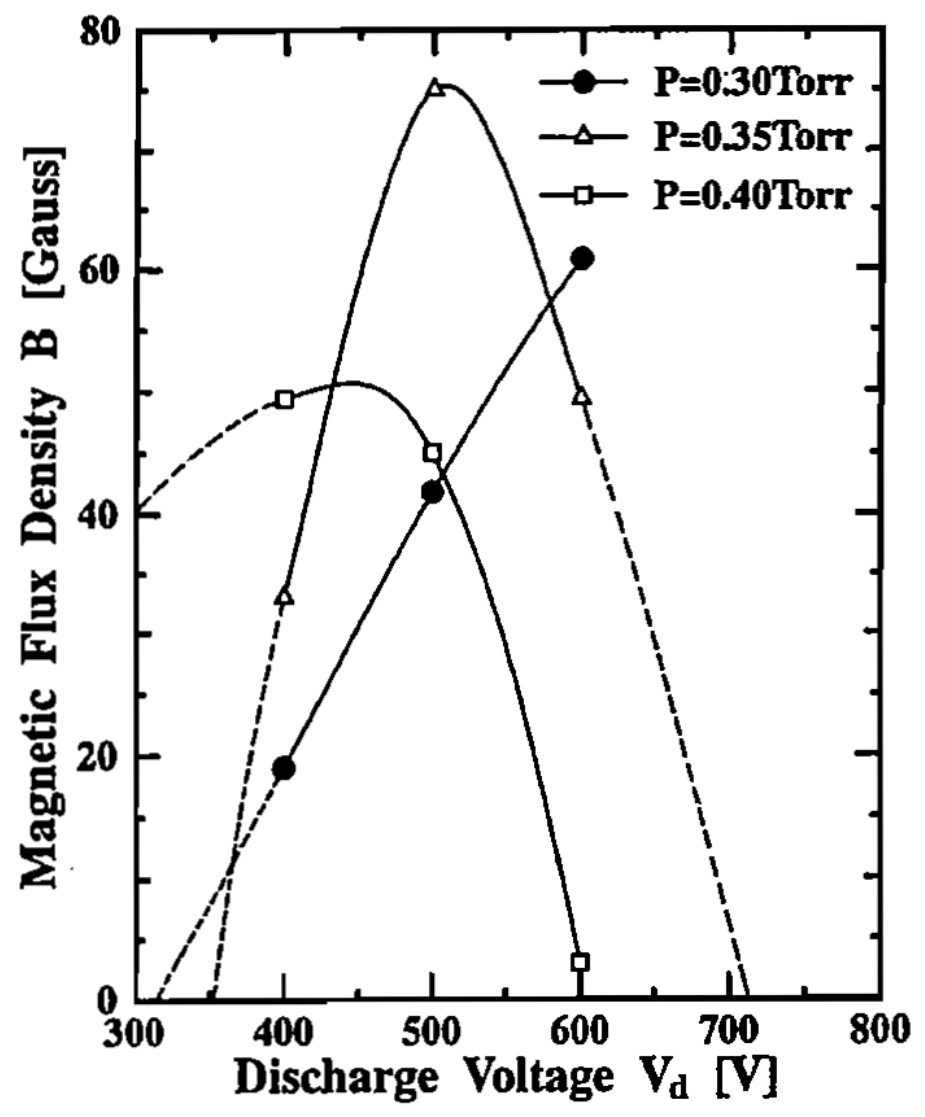

Figure 6. Dependence on discharge voltage of the magnetic flux density required to exclude silicon particles from the discharge space. 


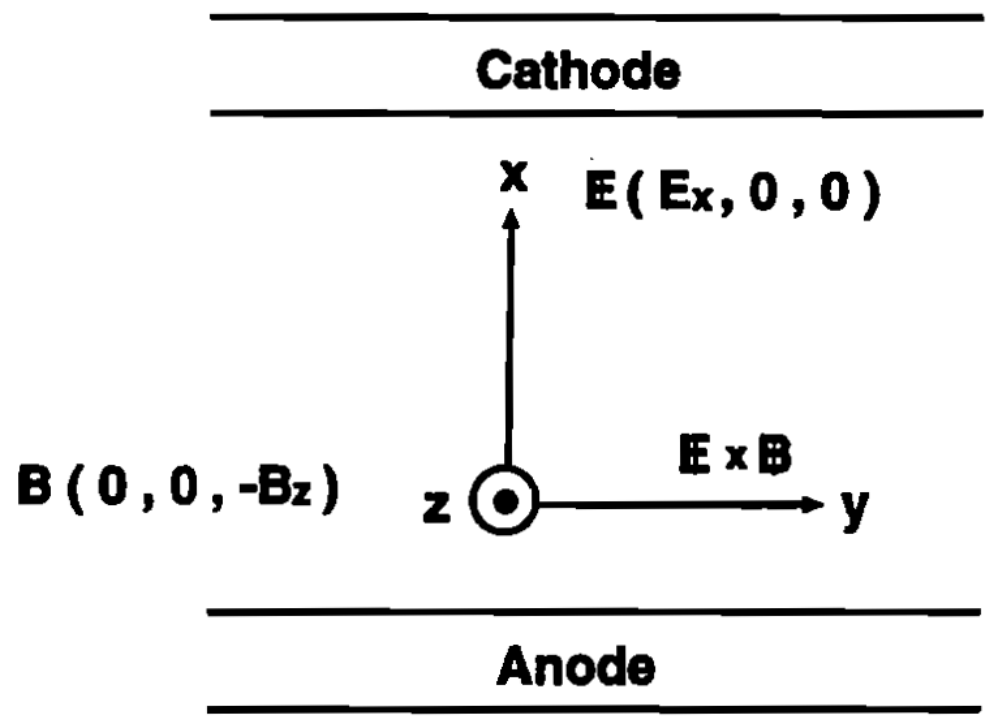

Figure 7. Calculation model in a DC dusty plasma in the presence of a crossed magnetic field. 
(a)

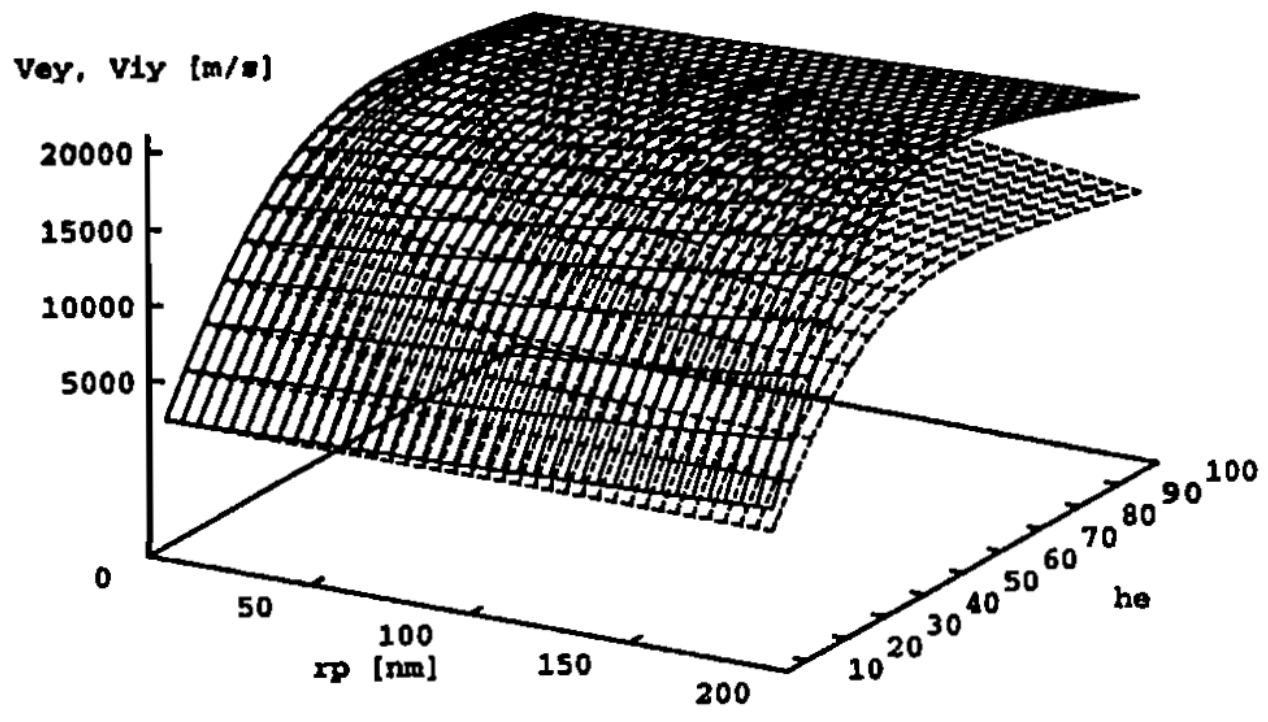

(b)

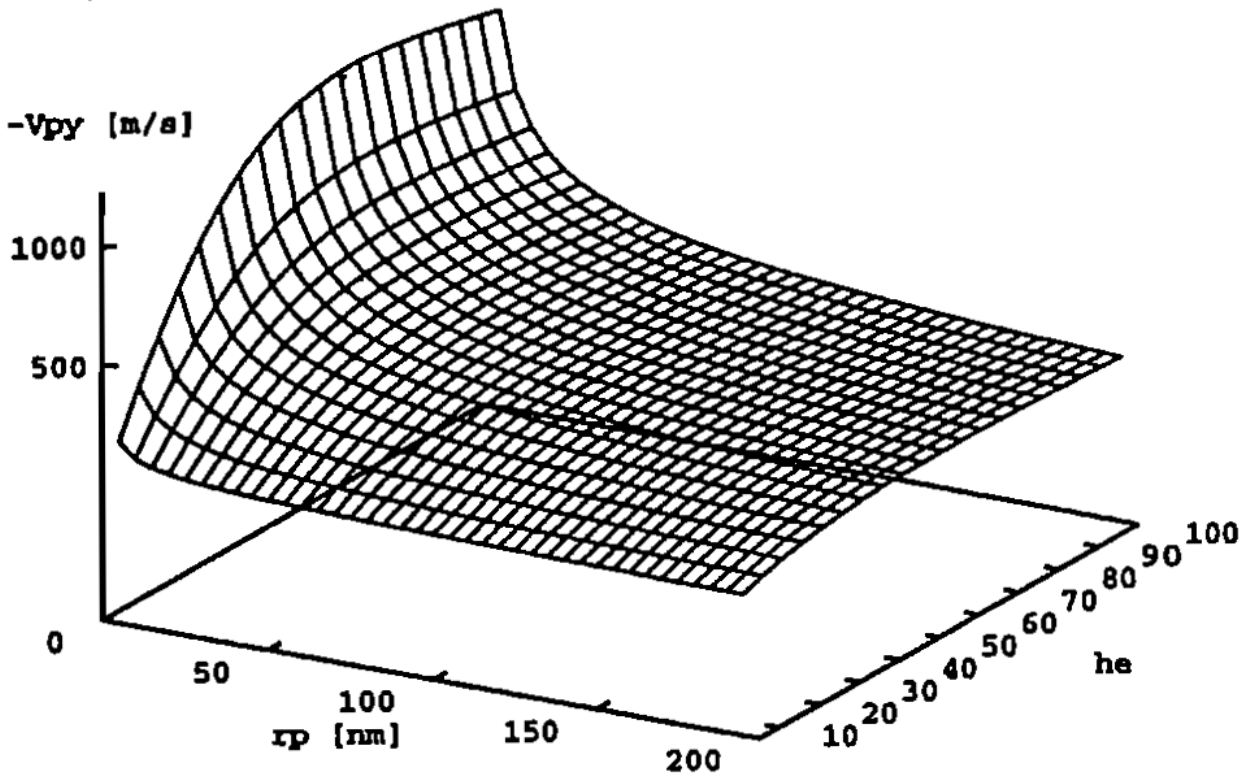

Figure 8. Typical calculation results of drift velocity of (a) electrons and ions and (b) negative ions (silicon particles) against particle radius $r_{p}$ and electron Hall parameter $h_{\theta}$. 